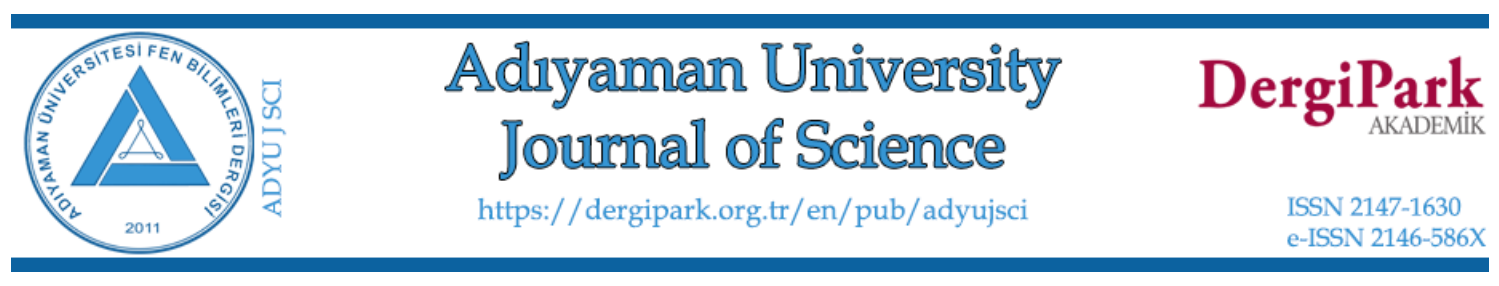

\title{
On $\Delta$-Uniform and $\Delta$-Pointwise Convergence on Time Scale
}

\author{
Mustafa Seyyit SEYYIDOĞLU ${ }^{1, *}$, Ayşe KARADAŞ² \\ ${ }^{1}$ Uşak University, Department of Mathematics, Uşak, Turkey \\ seyyit.seyyidoglu@usak.edu.tr,ORCID:0000-0001-9129-1373 \\ ${ }^{2}$ Uşak University, Institute of Science and Technology, Uşak, Turkey \\ aysekaradas9496@gmail.com,ORCID:0000-0000-0000-0000
}

$\begin{array}{lll}\text { Received: 29.03.2019 } & \text { Accepted: 03.03.2020 } & \text { Published: 25.06.2020 }\end{array}$

\begin{abstract}
In this article, we define the concept of $\Delta$-Cauchy, $\Delta$-uniform convergence and $\Delta$ pointwise convergence of a family of functions $\left\{f_{j}\right\}_{j \in \mathbb{J}}$, where $\mathbb{J}$ is a time scale. We study the relationships between these notions. Moreover, we introduced sufficient conditions for interchangeability $\Delta$-limitation with Riemann $\Delta$-integration or $\Delta$-differentiation. Also, we obtain the analogue of the well-known Dini's Theorem.
\end{abstract}

Keywords: $\Delta$-Convergence; $\Delta$-Cauchy; Statistical convergence.

\section{Zaman Skalası Üzerinde $\Delta$-Düzgün ve $\Delta$-Noktasal Yakınsaklık}

\section{$\ddot{O} z$}

Bu makalede \ bir zaman skalası olmak üzere, $\left\{f_{j}\right\}_{j \in \mathbb{J}}$ fonksiyon ailesi için $\Delta$-Cauchy, $\Delta$ düzgün yakınsaklık ve $\Delta$-noktasal yakınsaklık kavramları verilerek bu kavramlar arasındaki ilişkiler incelenmiştir. $\Delta$-limit ile Riemann $\Delta$-integrali ve $\Delta$-türevin yer değişme problemi araştırılarak Dini Teoreminin farklı bir versiyonu elde edilmiştir.

Anahtar Kelimeler: $\Delta$-Yakınsaklık; $\Delta$-Cauchy; İstatistiksel yakınsaklık. 


\section{Introduction and Preliminaries}

The time scale calculus was introduced in 1989 by German mathematician Stefan Hilger [1]. It is a unification of the theory of differential equations with that of difference equations. This theory was developed to a certain extent in [2] by Hilger.

The notion of statistical convergence for complex number sequences was introduced by Fast in [3]. Schoenberg gave some properties of this concept [4]. Fridy progressed with the statistically Cauchy and showed the equivalence of these concepts in [5].

In recent years, there are many studies based on the density function, which is defined on some subsets of time scale. For instance, first author and Tan [6] gave the notions of $\Delta$-Cauchy and $\Delta$-convergence of a function defined on time scale by using $\Delta$-density. The notion of $m$-and $(\lambda, m)$ - uniform density of a set and the concept of $m$ - and $(\lambda, m)$ - uniform convergence on a time scale were presented by Altin et al. [7]. Also, Altin et al. gave $\lambda$-statistical convergence on time scale and examined some of its features [8]. Some fundamental properties of Lacunary statistical convergence and statistical convergence on time scale investigated by Turan and Duman in [9].

Let $\mathcal{S}$ be the collection of all subsets of time scale $\mathbb{J}$ in the form of $[a, b)$, where $[a, a)=\emptyset$. Then $\mathcal{S}$ is a semiring on $\mathbb{J}$. The set function $m$ defined by $m([a, b))=b-a$ is a measure on $\mathcal{S}$. The outer measure $m^{*}: \mathcal{S} \rightarrow[0, \infty]$ generated by $m$ is defined by

$$
m^{*}(A):=\inf \left\{\sum_{n=1}^{\infty}\left(b_{n}-a_{n}\right): A \subset \bigcup_{n=1}^{\infty}\left[a_{n}, b_{n}\right)\right\} .
$$

The family of all $m^{*}$-measurable (it is also called $\Delta$-measurable) sets $\mathcal{M}=\mathcal{M}\left(m^{*}\right)$ is a $\sigma$ algebra and it is well known that from the measure theory the restriction of $m^{*}$ to $\mathcal{M}$, which we denote by $\mu_{\Delta}$, is a measure. This measure is called Lebesgue $\Delta$-measure on $\mathbb{J}$.

Definition 1. [5] Let $A \subset \mathbb{N}$, and

$$
A_{n}=\sum_{m \leq n, m \in A} 1
$$

The asymptotic density of $A$ is defined by $\delta(A)=\lim _{n} n^{-1} A_{n}$, which is also called natural density. The real number sequence $x=\left(x_{n}\right)$ is statistically convergent to $l$ if for each $\epsilon>0$, $\delta\left(\left\{n \in \mathbb{N}:\left|x_{n}-l\right| \geq \epsilon\right\}=0\right.$; in this case we write st-lim $x=l$.

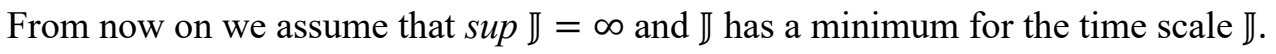


Definition 2. ( $\Delta$-Density) [6] Let $B$ be a subset of $\mathbb{J}$ such that $B \in \mathcal{M}$ and $a=\min \mathbb{\rrbracket}$. $\Delta$ density of $B$ in $\mathbb{J}$ is defined by

$$
\delta_{\Delta}(B):=\lim _{j \rightarrow \infty} \frac{\mu_{\Delta}(B \cap[a, j])}{\sigma(j)-a}
$$

provided that this limit exists.

A property of points of $\mathbb{J}$ is said to hold $\Delta$-almost everywhere (or $\Delta$-almost all $j \in \mathbb{J}$ ) if the set of points in $\mathbb{J}$ at which it fails to hold has zero $\Delta$-density. The expression $\Delta$-almost everywhere abbreviated to $\Delta$-a.e.

Definition 3. ( $\Delta$-Convergence) [6] If for every $\epsilon>0$, the inequality $|g(j)-l|<\epsilon$ holds $\Delta$-a.e. on $\mathbb{J}$, then $g: \mathbb{J} \rightarrow \mathbb{R}$ is called $\Delta$-convergent to $l \in \mathbb{R}$ (or has $\Delta$-limit). In this case we write $\Delta-\lim _{j \rightarrow \infty} f(j)=l$.

Definition 4. ( $\Delta$-Cauchy) [6] The function $g: \mathbb{I} \rightarrow \mathbb{R}$ is $\Delta$-Cauchy provided that for each $\epsilon>0$, there exist $K=K(\epsilon) \subset \mathbb{J}$ and $j_{0} \in \mathbb{J}$ such that $\delta_{\Delta}(K)=1$ and $\left|g(j)-g\left(j_{0}\right)\right|<\epsilon$ holds for all $j \in K$.

Note that the $\Delta$-density, $\Delta$-Cauchy and $\Delta$-Convergence coincide with the natural density, statistical Cauchy and statistical convergence respectively whenever $\mathbb{J}$ is the natural numbers.

\section{2. $\Delta$-Pointwise and $\Delta$-Uniform Convergence}

In this section, we will deal with the family of functions $\left\{f_{j}\right\}_{j \in \mathbb{J}}$ whose elements defined on any subset of real numbers.

Definition 5. ( $\Delta$-Pointwise Convergence) Let $B \subset \mathbb{R}$ and for each $j \in \mathbb{V}, f_{j}$ and $f$ be real valued functions on $B$. The family $\left\{f_{j}\right\}_{j \in \mathbb{V}}$ converges $\Delta$-pointwise to $f$ on $\mathrm{B}$, if for each given $\epsilon>$ 0 and $t \in B$, the inequality $\left|f_{j}(t)-f(t)\right|<\epsilon$ holds $\Delta$-a.e. on $\mathbb{J}$. This notion is abbreviated as $\left\{f_{j}\right\}_{j \in \mathbb{J}} \rightarrow f$ on $B$.

Definition 6. ( $\Delta$-Uniform Convergence) Let $B \subset \mathbb{R}$ and for each $j \in \mathbb{J}, f_{j}$ and $f$ be real valued functions on $B$. The family $\left\{f_{j}\right\}_{j \in \mathbb{J}}$ converges $\Delta$-uniformly to $f$ on $B$, if for each given $\epsilon>$ 0 , the inequality $\left|f_{j}(t)-f(t)\right|<\epsilon$ holds $\Delta$-a.e. on $\mathbb{J}$ and for all $t \in B$. In this case we write $\left\{f_{j}\right\}_{j \in \mathbb{J}} \rightrightarrows f$ on $B$. 
Definition 7. ( $\Delta$-Uniform Cauchy) Let $B \subset \mathbb{R}$ and $\left\{f_{j}\right\}$ be a family of real valued functions defined on $B$. The family $\left\{f_{j}\right\}_{j \in \mathbb{V}}, \Delta$-uniform Cauchy on $B$, if for all $\epsilon>0$ there exists a subset $K=K(\epsilon)$ of $\mathbb{J}$ and $j_{0} \in \mathbb{J}$ such that $\delta_{\Delta}(K)=1$ and $\left|f_{j}(t)-f_{j_{0}}(t)\right|<\epsilon$ for all $j \in \mathrm{K}$ and for all $t \in B$.

Example 8. Let $\mathbb{J}=[0, \infty)$ and $B \subset \mathbb{R}$. We denote the irrational and rational numbers in $[0, \infty)$ by $\mathbb{I}_{[0, \infty)}$ and $\mathbb{Q}_{[0, \infty)}$, respectively. We consider the functions $f_{j}: B \rightarrow \mathbb{R}(j \in \mathbb{I})$ defined as;

$$
f_{j}(t)= \begin{cases}\sin j t, & j \in \mathbb{Q}_{[0, \infty)} \\ 0, & j \in \mathbb{I}_{[0, \infty)}\end{cases}
$$

Since the set $\mathbb{Q}_{[0, \infty)}$ has zero density in $\mathbb{J}$, the density of $\mathbb{I}_{[0, \infty)}$ is one. Hence, $\left\{f_{j}\right\}_{j \in \mathbb{J}} \rightrightarrows f=0$ on $B$.

It is easily seen that $\Delta$-uniform convergence implies $\Delta$-pointwise convergence, but the converse is not always true as we can see from the following counter-examle.

Example 9. Let $\mathbb{J}=[1, \infty)$ and $j \in \mathbb{J}$. Consider the functions $f_{j}:[0, \infty) \rightarrow \mathbb{R}$ defined as;

$$
f_{j}(t)=\left\{\begin{array}{ll}
\frac{t}{j}, & j \in \mathbb{Q}_{[1, \infty)} \\
0, & j \in \mathbb{I}_{[0, \infty)}
\end{array} .\right.
$$

Although $\left\{f_{j}\right\}_{j \in \mathbb{J}}$ is $\Delta$-pointwise convergent to $f=0$, it is not $\Delta$-uniform convergent.

The proof of the following theorem is clear.

Theorem 10. Let $\left(f_{n}\right)_{n \in \mathbb{N}}$ be a sequence of real valued functions defined on $B \subset \mathbb{R}$. If $\left(f_{n}\right)_{n \in \mathbb{N}}$ converges uniformly (pointwise) to $f$, then $\left\{f_{n}\right\}_{n \in \mathbb{N}}$ converges $\Delta$-uniformly ( $\Delta$-pointwise) to $f$.

Theorem 11. Let $\left\{f_{j}\right\}_{j \in \mathbb{J}}$ be a family of real valued functions defined on $B \subset \mathbb{R}$. If $\left\{f_{j}\right\}_{j \in \mathbb{I}} \rightarrow f$ on $B$, then $\left\{f_{j}\right\}_{j \in \mathbb{I}} \rightrightarrows f$ on $B$ if and only if

$$
\Delta-\limsup _{j \rightarrow \infty}\left|f_{j}(t)-f(t)\right|=0
$$

Theorem 12. Let $\left\{f_{j}\right\}_{j \in \mathbb{J}}$ be a family of real valued functions defined on $B \subset \mathbb{R} .\left\{f_{j}\right\}_{j \in \mathbb{I}} \rightrightarrows$ $f$ on $B$ if and only if it is $\Delta$-uniform Cauchy on $B$.

Proof. Necessity is obvious. Let $\left\{f_{j}\right\}_{j \in \mathbb{J}}$ be $\Delta$-uniform Cauchy on $B$. For a given $\epsilon>0$ there exists $j_{0} \in \mathbb{J}$ and $K \subset \mathbb{J}$ such that $\delta_{\Delta}(K)=1$, the inequality 


$$
\left|f_{j}(t)-f_{j_{0}}(t)\right|<\frac{\epsilon}{2}
$$

holds for all $j \in K$ and $t \in B$. Let $g_{t}: \mathbb{J} \rightarrow \mathbb{R}$ defined by $g_{t}(j)=f_{j}(t)$ for each $t \in B$. For each fixed $t$

$$
\left|g_{t}(j)-g_{t}\left(j_{0}\right)\right|=\left|f_{j}(t)-f_{j_{0}}(t)\right|<\epsilon
$$

holds $\Delta$-a.e. on $\mathbb{J}$. Therefore, the functions $g_{t},(t \in B)$ are $\Delta$-Cauchy. These functions have $\Delta$ limit. Let $f(t)=\Delta$ - $\lim _{j \rightarrow \infty} g_{t}(j)$. As $j \rightarrow \infty$, the $\Delta$-limit of (1) yields

$$
\left|f(t)-f_{j_{0}}(t)\right| \leq \frac{\epsilon}{2}
$$

In view of inequalities (1) and (2), one can get

$$
\left|f_{j}(t)-f(t)\right| \leq\left|f_{j}(t)-f_{j_{0}}(t)\right|+\left|f_{j_{0}}(t)-f(t)\right|<\epsilon,
$$

for all $j \in K$ and for all $t \in B$.

Theorem 13. Let $\mathbb{T}$ and $\mathbb{J}$ be two time scales and $[\alpha, \beta] \subset B \subset \mathbb{T}$. If $f_{j} \in C_{r d}(B, \mathbb{R}):=$ $\{f \mid f: B \rightarrow \mathbb{R}$ is $r d$ - continuous $\}$ for all $j \in \mathbb{J}$, and $\left\{f_{j}\right\}_{j \in \mathbb{J}} \rightrightarrows f$, then $f \in C_{r d}(B, \mathbb{R})$ and

$$
\Delta-\lim _{j \rightarrow \infty} \int_{\alpha}^{\beta} f_{j}(t) \Delta t=\int_{\alpha}^{\beta} f(t) \Delta t
$$

Proof. Let any positive $\epsilon$ be given. In accordance with $\Delta$-uniform convergence, the time scale $\mathbb{J}$ has a subset $K$ such that $\delta_{\Delta}(K)=1$ and the inequality

$$
\left|f_{j}(t)-f(t)\right|<\frac{\epsilon}{3}
$$

holds for all $j \in K$ and for all $t \in B$.

Let $j_{0} \in K$ and $t_{0} \in B$ are arbitrary. We consider two cases. In the first case we assume that $t_{0}$ is left-dense. From rd-continuity of $f_{j_{0}}$, we can find $\delta>0$ such that

$$
\left|f_{j_{0}}(\xi)-f_{j_{0}}(\eta)\right|<\frac{\epsilon}{3}
$$

for any $\xi, \eta \in\left(t_{0}-\delta, t_{0}\right)$. If $t_{n} \rightarrow t_{0}^{-}$as $n \rightarrow \infty$, then there exists natural number $n_{0}$ such that $n, m>n_{0}$ imply $t_{m}, t_{n} \in\left(t_{0}-\delta, t_{0}\right)$ and

$$
\left|f_{j_{0}}\left(t_{n}\right)-f_{j_{0}}\left(t_{m}\right)\right|<\frac{\epsilon}{3} .
$$


Hence, for $m, n>n_{0}$, we have

$$
\begin{aligned}
\mid f\left(t_{n}\right)- & f\left(t_{m}\right)|=| f\left(t_{n}\right)-f_{j_{0}}\left(t_{n}\right)+f_{j_{0}}\left(t_{n}\right)-f_{j_{0}}\left(t_{m}\right)+f_{j_{0}}\left(t_{m}\right)-f\left(t_{m}\right) \mid \\
\leq & \left|f\left(t_{n}\right)-f_{j_{0}}\left(t_{n}\right)\right|+\left|f_{j_{0}}\left(t_{n}\right)-f_{j_{0}}\left(t_{m}\right)\right| \\
& \quad+\left|f_{j_{0}}\left(t_{m}\right)-f\left(t_{m}\right)\right| \\
< & \epsilon .
\end{aligned}
$$

Therefore, the function $f$ has finite left-sided limit at $t_{0}$.

In the second case we assume that $t_{0}$ is right-dense. Then all functions $f_{j}$ are continuous at $t_{0}$. If $t_{n} \rightarrow t_{0}$ as $n \rightarrow \infty$, then there exists natural number $n_{0}$ such that $n, m>n_{0}$ imply $t_{m}, t_{n} \in$ $\left(t_{0}-\delta, t_{0}+\delta\right)$ and (3-4) holds. This is implies continuity of $f$ at $t_{0}$. Therefore, $f$ is Riemann $\Delta$ integrable on every subinterval $[\alpha, \beta] \subset B$. So, we obtain the inequality

$$
\left|\int_{\alpha}^{\beta} f_{j}(t) \Delta t-\int_{\alpha}^{\beta} f(t) \Delta t\right| \leq \int_{\alpha}^{\beta}\left|f_{j}(t)-f(t)\right| \Delta t<\frac{\epsilon}{3}(\beta-\alpha),
$$

for every $j \in K$ that completes our proof.

Theorem 14. Let $\mathbb{T}$ and $\mathbb{J}$ be two time scales and $[\alpha, \beta] \subset \mathbb{T}$. Suppose that the functions

$$
f_{j}:[\alpha, \beta] \rightarrow \mathbb{R} \quad(j \in \mathbb{J})
$$

satisfies the following conditions on $[\alpha, \beta]$ :

1. $f_{j}$ has Hilger derivative and its Hilger derivative $f_{j}^{\Delta}$ is rd-continuous,

2. $\left\{f_{j}\right\}_{j \in \mathbb{J}} \rightarrow f$,

3. $\left\{f_{j}^{\Delta}\right\}_{j \in \mathbb{J}} \rightrightarrows g$.

Then $f$ has Hilger derivative on $[\alpha, \beta]$ and $f^{\Delta}(t)=g(t)$ for all $t \in[\alpha, \beta]$.

Proof. $g$ is rd-continuous on $[\alpha, \beta]$ by Theorem 13 and so $g$ is Riemann $\Delta$-integrable on this interval. By the help of Theorem 13, we have

$$
\int_{\alpha}^{t} g(s) \Delta s=\Delta-\lim _{j \rightarrow \infty} \int_{\alpha}^{t} f_{j}^{\Delta} f(s) \Delta s=\Delta-\lim _{j \rightarrow \infty}\left(f_{j}(t)-f_{j}(\alpha)\right)=f(t)-f(\alpha),
$$


for all $t \in[\alpha, \beta]$. Since the left hand-side of the last equality has Hilger derivative, the right handside also has, and it follows that $f^{\Delta}(t)=g(t)$ for all $t \in[\alpha, \beta]$.

Theorem 15. (Dini's Theorem) Let $X$ be a compact metric space. Let $f: X \rightarrow \mathbb{R}$ be a continuous function and the functions $f_{j}: X \rightarrow \mathbb{R},(j \in \mathbb{J})$ are continuous for $\Delta$-almost all $\mathbb{J}$. If the following two conditions are satisfied:

1. $\left\{f_{j}\right\}_{j \in \mathbb{I}} \rightarrow f$ on $X$,

2. $f_{j}(x) \leq f_{i}(x)$ for all $x \in X$ and $\Delta$-almost all $i, j \in \mathbb{J}$ such that $i<j$,

then $\left\{f_{j}\right\}_{j \in \mathbb{J}} \rightrightarrows f$ on $X$.

Proof. There exists a subset $K_{1} \subset \mathbb{J}$ with $\Delta$-density 1 . Moreover, for each $j \in K_{1}$ the functions $f_{j}$ are continuous, and

$$
f_{j}(x) \leq f_{i}(x) \text { for all } x \in X
$$

holds for all $i, j \in K_{1}$ such that $i<j$. For each $j \in K_{1}$, define $g_{j}=f_{j}-f$. Then $\left\{g_{j}\right\}_{j \in K_{1}}$ is a family of continuous functions on the compact metric space $X$ that converges $\Delta$-pointwise to 0 . Furthermore,

$$
0 \leq g_{j}(x) \leq g_{i}(x)
$$

for all $x \in X$ and $i, j \in K_{1}$ such that $i<j$.

Let $\epsilon>0$ and define

$$
G_{j}=\left\{x \in X: g_{j}(x)<\epsilon\right\}, \quad\left(j \in K_{1}\right)
$$

Since $g_{j}$ is continuous, then $G_{j}$ is an open set and $G_{i} \subset G_{j}$ for each $i, j \in K_{1}$ such that $i<j$.

Let $x_{0} \in X$ be arbitrary. Since $\Delta-\lim _{j \rightarrow \infty} g_{j}\left(x_{0}\right)=0$, then there exists a subset $K_{2} \subset \mathbb{J}$ such that $\delta_{\Delta}\left(K_{2}\right)=1$ and the inequality $\left|g_{j}\left(x_{0}\right)\right|<\epsilon$ holds for all $j \in K_{2}$. If we set $K=K_{1} \cap K_{2}$ then $\delta_{\Delta}(K)=1$ and $g_{j}\left(x_{0}\right)=\left|g_{j}\left(x_{0}\right)\right|<\epsilon$ for all $j \in K$. Thus $x_{0} \in G_{j}$ for all $j \in K$, and thus, we have

$$
X=\bigcup_{j \in K} G_{j}
$$


Since $K$ is compact and $G_{i} \subset G_{j}$ when $i<j$, then there is a $j_{0} \in K$ with $G_{j_{0}}=X$. Then we have $G_{j}=X$ for all $j \in K$ such that $j>j_{0}$. This implies that $f_{j}(x)-f(x)=g_{j}(x)<\epsilon$ for all $x \in X$ and $j \in K$ such that $j>j_{0}$. Consequently, $\left\{f_{j}\right\}_{j \in \mathbb{J}} \rightrightarrows f$ on $X$.

\section{References}

[1] Hilger, S., Ein maßkettenkalkül mit anwendung auf zentrumsmannigfaltigkeiten, $\mathrm{PhD}$ Thesis, 1989.

[2] Hilger, S., Analysis on measure chains-a unified approach to continuous and discrete calculus, Results in Mathematics 18, 1-2, 18-56, 1990.

[3] Fast, H., Sur la convergence statistique, Colloquium Mathematicae, 2(3-4), 1951.

[4] Schoenberg, I.J,.The integrability of certain functions and related summability methods, The American Mathematical Monthly, 66(5), 361-775, 1959.

[5] Fridy, J.A., On statistical convergence, Analysis,5(4), 301-314, 1985.

[6] Seyyidoğlu, M.S., Tan, N.Ö,. A note on statistical convergence on time scale, Journal of Inequalities and Applications, 2012(1), 219, 2012.

[7] Altın, Y., Koyunbakan, H., Y1lmaz, E., Uniform statistical convergence on time scales, Journal of Applied Mathematics, vol. 2014, 6 pages, 2014.

[8] Yilmaz, E., Altın, A., Koyunbakan, H., $\lambda$-Statistical convergence on time scales, Dynamics of Continuous, Discrete and Impulsive Systems Series A: Mathematical Analysis, 23, 69-78, 2016.

[9] Ceylan, T., Duman, O., Fundamental Properties of Statistical Convergence and Lacunary Statistical Convergence on Time Scales, Filomat, 31(14), 2017. 